GT2008-50119

\title{
ON THE ROLE OF THE DETERMINISTIC AND CIRCUMFERENTIAL STRESSES IN THROUGHFLOW CALCULATIONS
}

\author{
J.-F. Simon \\ Techspace Aero \\ Safran Group \\ Route de Liers 121 \\ B-4041 Milmort, Belgium \\ Email: jsimon@techspace-aero.be
}

\author{
O. Léonard \\ University of Liège \\ Turbomachinery Group \\ Chemin des Chevreuils 1 \\ B-4000 Liège, Belgium \\ Email: o.leonard@ulg.ac.be
}

\section{ABSTRACT}

This paper presents a throughflow analysis tool developed in the context of the average-passage flow model elaborated by Adamczyk. The Adamczyk's flow model describes the 3-D timeaveraged flow field within a blade row passage. The set of equations that governs this flow field is obtained by performing a Reynolds averaging, a time averaging and a passage-to-passage averaging on the Navier-Stokes equations. The throughflow level of approximation is obtained by performing an additional circumferential averaging on the 3-D average-passage flow.

The resulting set of equations is similar to the 2-D axisymmetric Navier-Stokes equations but additional terms resulting from the averages show up : blade forces, blade blockage factor, Reynolds stresses, deterministic stresses, passage-to-passage stresses and circumferential stresses. This set of equations represents the ultimate throughflow model provided that all stresses and blade forces can be modeled. The relative importance of these additional terms is studied in the present contribution.

The stresses and the blade forces are determined from 3-D steady and unsteady databases (a low speed compressor stage and a transonic turbine stage) and incorporated in a throughflow model based on the axisymmetric Navier-Stokes equations. A good agreement between the throughflow solution and the averaged 3-D results is obtained. These results are also compared to those obtained with a more "classical" throughflow approach based on a Navier-Stokes formulation for the endwall losses, correlations for profile losses and a simple radial mixing model assuming turbulent diffusion.

\section{NOMENCLATURE}

$b$ blockage factor

$f_{b}$ inviscid blade force

$f_{v}$ viscous blade force

$h, H$ enthalpy, total enthalpy

$k$ kinetic energy of the fluctuations

$p, p^{0}$ pressure, total pressure

$q$ heat flux

$s$ entropy (relative to inlet conditions)

$T^{0}$ total temperature

$V$ absolute velocity

$x, r, \theta$ axial,radial and circumferential coordinates

$\alpha$ absolute flow angle

$\rho$ density

$\tau$ viscous stress

Operators

- averaging operator

$\sim$ Favre averaging operator

Subscripts

$x, r, \theta$ axial, radial and circumferential components

$R$ relative to a rotor

$S$ relative to a stator Superscripts

' unsteady non-deterministic fluctuation

" unsteady deterministic fluctuation

'I' aperiodic fluctuation

'III' circumferential fluctuation 


\section{INTRODUCTION}

The throughflow level of approximation still remains an important tool for designing turbomachines [10] even though 3-D calculations are used more and more early in the design process. Throughflow codes are mainly used at the preliminary design stage for specifying the target aerodynamic performances to be achieved by the blading. They can be used either in the design mode, where the angular momentum and/or the total conditions are prescribed and the flow angles are sought, either in the analysis mode, where a known machine geometry is analyzed for its performance. The throughflow models are also used to exploit experimental results or to couple single blade row calculations in order to compute the flow field inside a multistage machine [4].

Unfortunately, these models heavily rely on empirical inputs, such as profile losses correlations or end-wall loss models. They can accurately predict the flow field inside a turbomachine provided that the design parameters are not too far from those of the reference machines that were used to calibrate the throughflow model. This approach has shown to be efficient but lacks of generality.

The most widespread throughflow method is certainly the streamline curvature method (SLC). In 1980, Spuur has proposed another approach based on the Euler equations. This approach has only started to retain attention in the 1990. Recent works using this approach can be found in [5] and [22]. The methods based on the Euler equations present some interesting features and eliminate some of the drawbacks of the streamline curvature approach, such as a shock capturing property or a natural unsteady capibility with the generally adopted time-marching technique.

However the treatment of the annulus endwalls is probably the major concern for the throughflow models based on the SLC method as well as the ones based on the Euler equations. A common practice is to introduce an aerodynamic blockage - equivalent to the displacement thickness of the endwall boundary layers - which corrects the mass flow in order to obtain the correct level of velocity in the core flow. The blockage factor is a very sensitive quantity relying on empiricism for which a misprediction can lead to a mismatch of the stages. Another solution is to perform a separate boundary layer calculation. However, it is recognized that the use of the boundary layer theory for computing the endwall flows inside a turbomachine (especially in a compressor) is inappropriate [9], [14].

Recently another solution has been proposed by the authors with a throughflow model directly based on the Navier-Stokes equations. It is able to resolve the viscous flow on the annulus endwalls and the corresponding aerodynamic blockage. It can also capture 2-D recirculations. The $\theta$-averaged Navier-Stokes equations are solved by a finite volume technique. By including more physics in the model, less empiricism is needed and a more general method can be devised. Details of this model as well as the numerical techniques used can be found in [17] and [18].
In the present contribution, the authors propose another step toward less empiricism in throughflow calculations with a highorder throughflow method. This model is based on the Adamczyk cascade of averaging procedure [1].

Adamczyk addresses the 3-D unsteady and turbulent flow field via several averaging operations. The first one is the well known Reynolds averaging which eliminates the effects of the turbulence, leaving a deterministic unsteady flow. The second one is a time averaging removing the remaining effects of the unsteadiness due to the movement of the rotor blades with respect to the stator ones. Finally an aperiodic averaging eliminates the aperiodicity of the flow due to the blade indexing. The resulting flow field is steady and periodic but it incorporates the mean effects of the turbulence, the unsteadiness and the aperiodicity. The equations associated to this flow show different unknown terms bringing the aforementioned effects. These terms are the Reynolds stresses, the deterministic stresses, the passageto-passage stresses and the blade forces. These equations, which have been rigorously obtained, are the so-called average-passage equations and describe the steady flow field inside a blade row embedded in a multistage environment.

In this contribution a subsequent step is performed by circumferentially averaging the average-passage equations in order to obtain an axisymmetric representation of the flow. These equations are rigorously obtained and contain the effects of the non-axisymmetry of the flow through circumferential stresses and blade forces. This set of equations represents the ultimate throughflow model. The sole assumptions are those prevailing to the establishment of the Navier-Stokes equations. However these throughflow equations present several unknown terms which have to be modelled or closed. This tremendous task is far beyond the scope of this paper. In the present contribution, the relative importance of the different terms of such a throughflow calculation is studied as well as the benefit brought by this model compared to more classical throughflow models.

These analysis are performed with the help of 2-D and 3-D test-cases. The development of a boundary layer over a flat plate and the spreading of a wake allow to highlight the main properties of the averaged equations and to perform a first attempt to evaluate the relative importance of the different unknowns brought by the averaging process. The high-order throughflow model is also applied to two turbomachine test-cases, a low speed compressor stage and a transonic turbine stage. These test-cases reside in 3-D steady and unsteady numerical simulations from which the different terms needed for the closure of the throughflow equations are extracted. They allow to further study the high-order throughflow model and to show the improvements compared to a classical throughflow. 


\section{CIRCUMFERENTIAL-AVERAGED EQUATIONS}

The average-passage set of equations of Adamczyk is obtained by successively averaging the unsteady Navier-Stokes equations on an ensemble of realizations - in the sense of Reynolds - on time and on the passages of a given blade row. This triple averaging procedure brings the mean effects of the turbulence, the unsteadiness and the aperiodicity on the steady flow field inside a blade row embedded in a multistage configuration. These effects appear as additional terms in the conservation equations, namely Reynolds stresses, deterministic stresses, passage-to-passage stresses, blade blockage factors and blade forces. For example, for a given stator $j$ embedded in a multistage machine, the resulting axial momentum equation is written as follows:

$$
\begin{aligned}
\frac{1}{b_{j}} \frac{\partial b_{j}\left(\bar{\rho} \widetilde{V}_{x} \widetilde{V}_{x}+\bar{p}\right)}{\partial x}+\frac{1}{r b_{j}} \frac{\partial r b_{j}\left(\bar{\rho} \widetilde{V}_{r} \widetilde{V}_{x}\right)}{\partial r}+\frac{1}{r b_{j}} \frac{\partial b_{j}\left(\bar{\rho} \widetilde{V}_{\theta} \widetilde{V}_{x}\right)}{\partial \theta}+ \\
\quad=\frac{1}{b_{j}} \frac{\partial b_{j}\left(\overline{\tau_{x x}}-\overline{\rho V_{x}^{\prime} V_{x}^{\prime}}-\overline{\rho V_{x}^{\prime \prime} V_{x}^{\prime \prime}}-\overline{\rho V_{x}^{\prime \prime \prime} V_{x}^{\prime \prime \prime}}\right)}{\partial x} \\
+\frac{1}{r b_{j}} \frac{\partial r b_{j}\left(\overline{\tau_{r x}}-\overline{\rho V_{r}^{\prime} V_{x}^{\prime}}-\overline{\rho V_{r}^{\prime \prime} V_{x}^{\prime \prime}}-\overline{\rho V_{r}^{\prime \prime \prime} V_{x}^{\prime \prime \prime}}\right)}{\partial r} \\
+\frac{1}{r b_{j}} \frac{\partial b_{j}\left(\overline{\tau_{\theta x}}-\overline{\rho V_{\theta}^{\prime} V_{x}^{\prime}}-\overline{\rho V_{\theta}^{\prime \prime} V_{x}^{\prime \prime}}-\overline{\rho V_{\theta}^{\prime \prime \prime} V_{x}^{\prime \prime \prime}}\right)}{\partial \theta} \\
+\overline{f_{b x}^{R}}+\overline{f_{v x}^{R}}+\overline{f_{b x}^{S j}}+\overline{f_{v x}^{S j}}
\end{aligned}
$$

where $\overline{\rho V_{i}^{\prime} V_{j}^{\prime}}$ are the Reynolds stresses (which have been time and passage-to-passage averaged), $\overline{\rho V_{i}^{\prime \prime} V_{j}^{\prime \prime}}$ are the deterministic stresses (which have been passage-to-passage averaged) and $\overline{\rho V_{i}^{\prime \prime \prime} V_{j}^{\prime \prime \prime}}$ are the aperiodic stresses. The inviscid and viscous blade forces $\overline{f_{b x}^{R}}$ and $\overline{f_{v x}^{R}}$ originate from the rotors while the inviscid and viscous blade forces $\overline{f_{b x}^{S j}}$ and $\overline{f_{v x}^{S j}}$ are due to the stators others than the one under consideration. $b_{j}$ is the blade blockage factor. It is due to the blade thickness of all the blade rows except the one under consideration.

To simplify the notations, only one averaging symbol is used for the averaged quantities, e.g. $\bar{p}$ is used instead of $\overline{\bar{p}}$. For rigorous notations and details on the average-passage set of equations, see [1]. A great introduction to the flow physics linked to the average-passage equations can be found in [2] together with results from several simulations performed on both high and lowspeed compressors and turbines.

As it is the throughflow level of approximation which is of interest here, an additional (circumferential) averaging operation is performed on the average-passage system of equations. Here again, this procedure brings additional terms in the set of equations. The circumferential average can be defined with the help of a gate function in the same way as the time average defined by Adamczyk [1].

The circumferential average performed on the averagepassage flow field of the $j^{\text {th }}$ blade row is defined as follows:

$$
\bar{f}=\frac{\frac{1}{\Delta \theta} \int_{0}^{\Delta \theta} F(x, r, \theta) f(x, r, \theta) d \theta}{\frac{1}{\Delta \theta} \int_{0}^{\Delta \theta} F(x, r, \theta) d \theta}
$$

$\Delta \theta$ is equal to the pitch of the blade row under consideration, i.e. $2 \pi / N$ where $N$ is the blade count. $F$ is a gate function. Its value is unity when the point located at $(x, r, \theta)$ lies inside the flow field. It is equal to zero when the point lies inside the blade. The integral of the gate function over the pitch is equal to the blade blockage factor $b$ :

$$
b=\frac{1}{\Delta \theta} \int_{0}^{\Delta \theta} F(x, r, \theta) d \theta
$$

The flow field is next decomposed in an axisymmetric part and in a circumferential fluctuation part:

$$
f(x, r, \theta, t)=\bar{f}(x, r, \theta)+f^{\prime \prime \prime \prime}(x, r, \theta, t)
$$

The Favre average is used for the velocities and the total flow properties. The rule for averaging the partial derivative is the following:

$$
\frac{\overline{\partial f}}{\partial x_{i}}=\frac{1}{b} \frac{\partial b \bar{f}}{\partial x_{i}}-\frac{N}{2 \pi b}\left(f_{1} \frac{\partial \theta_{1}}{\partial x_{i}}-f_{2} \frac{\partial \theta_{2}}{\partial x_{i}}\right)
$$

with

$$
f_{1}=f\left(x, r, \theta=\theta_{1}\right) \quad \text { and } \quad f_{2}=f\left(x, r, \theta=\theta_{2}\right)
$$

where $\theta_{1}$ and $\theta_{2}$ are the locations of the blade sides in their order of appearance when rotating in the $\theta$ direction. They are measured with respect to the blade mean line. The additional term in the right-hand-side of equation (5) is only non-zero for quantities that are not equal to zero on the blade walls, i.e. the pressure, the viscous shear stress or the heat flux. This term is responsible for the inviscid and viscous blade forces.

As an example, the resulting axial momentum equation is 
given hereafter; all details of the derivation can be found in [19].

$$
\begin{aligned}
\frac{1}{b} \frac{\partial b\left(\bar{\rho} \widetilde{V}_{x} \widetilde{V}_{x}+\bar{p}\right)}{\partial x}+\frac{1}{r b} \frac{\partial r b\left(\bar{\rho} \widetilde{V}_{r} \widetilde{V}_{x}\right)}{\partial r} \\
\quad=\frac{1}{b} \frac{\partial b\left(\overline{\tau_{x x}}-\overline{\rho V_{x}^{\prime} V_{x}^{\prime}}-\overline{\rho V_{x}^{\prime \prime} V_{x}^{\prime \prime}}-\overline{\rho V_{x}^{\prime \prime \prime} V_{x}^{\prime \prime \prime}}-\overline{\rho V_{x}^{\prime \prime \prime \prime} V_{x}^{\prime \prime \prime \prime}}\right)}{} \\
+\frac{1}{r b} \frac{\partial r b\left(\overline{\tau_{r x}}-\overline{\rho V_{r}^{\prime} V_{x}^{\prime}}-\overline{\rho V_{r}^{\prime \prime} V_{x}^{\prime \prime}}-\overline{\rho V_{r}^{\prime \prime \prime} V_{x}^{\prime \prime \prime}}-\overline{\rho V_{r}^{\prime \prime \prime \prime} V_{x}^{\prime \prime \prime \prime}}\right)}{\partial r} \\
+\overline{f_{b x}^{R}}+\overline{f_{v x}^{R}}+\overline{f_{b x}^{S j}}+\overline{f_{v x}^{S j}}+f_{b x}^{j}+f_{v x}^{j}
\end{aligned}
$$

Compared to the average-passage equation (1) the $\theta$-derivatives disappear but additional terms appear:

- The term $\overline{\rho V_{i}^{\prime \prime \prime \prime} V_{j}^{\prime \prime \prime \prime}}$ is the circumferential stress. It represents the transport of momentum between the 3-D periodic and the axisymmetric flow fields.

- The blockage factor $b$ is due to the geometrical blockage resulting from the blades thickness.

- The momentum equations contain the blade forces $f_{b}^{j}$ and $f_{v}^{j}$. These terms are due to the pressure field and the shear stresses acting on the wall surfaces of the blade row $j$.

The obtained set of equations has been rigorously established from the 3-D instantaneous Navier-Stokes equations and represents the ultimate throughflow model. The equations are expressed in terms of the averaged conservative variables. Additionally to these primary unknowns, a large number of other unknown terms appear, which have to be modelled in order to solve the throughflow problem. For example for the momentum equations, the additional terms are the averaged viscous (laminar) stresses, the Reynolds stresses, the deterministic stresses, the passage-to-passage stresses, the circumferential stresses, the inviscid and the viscous blade forces.

If all these terms could be modelled adequately, one would obtain a perfect throughflow model able to predict the mean steady axisymmetric effect of the wake chopping phenomenon, the effect of the radial mixing or the mean effect of the blade clocking. Establishing a closure for that model is far beyond the scope of this work. In the present contribution, the focus will rather be put on the study of the system of equations which present certain peculiarities. Answers to the two following questions will also be sought:

1. What are the relative importance of the different terms and, from there, the terms that are worth to be modelled ?

2. What is the benefit of such a model compared to a classical throughflow model ?

In a 1966 paper [20], Smith addressed the first objective, with an analysis of the relative importance of the different terms present in the radial equilibrium equation. However, due to the unavailability of 3-D Navier-Stokes simulations at that time but also due to the less powerful throughflow model used (radial equilibrium vs 3-D Naviers-Stokes circumferentially averaged), several assumptions were made in his analysis. Noteworthy, a linear evolution of the quantities with $\theta$ between the pressure and suction sides and a basically inviscid formulation (with however an addition of a friction force). The Smith's conclusion concerning the circumferential stresses was that they were negligible although he recognized they could be important for machines with higher loading. With nowaday's tools, but also with more modern compressors and turbines, it is worthwhile analysing again this problem.

The two objectives described above will be achieved here with the help of 3-D steady and unsteady simulations, from which the different contributions to the momentum and energy equations will be extracted to feed a throughflow model. These studies on real turbomachine test-cases will be presented in a later section, after a preliminary study on simple 2-D test-cases modelled by a 1-D set of averaged equations. In the following, the Reynolds stresses will be modelled by an algebraic turbulence model and the passage-to-passage closure will not be addressed, as single-stage test-cases are considered.

\section{ONE-DIMENSIONAL STUDIES}

The purpose of this section is to present a preliminary study of the different contributions arising from the circumferential averaging process as well as to establish a hierarchy of these contributions. The study will therefore focus on the circumferential closure only; the deterministic stresses or the other terms arising from the time-averaging procedure will not be considered here.

It seems obvious that the inviscid blade force will take a great importance in the momentum and energy balances. However, the relative importance of the circumferential and viscous stresses is less clear. To help evaluating this relative importance, two simple numerical experiments are presented. These experiments consist in averaging a 2-D flow and its associated equations, obtaining a 1-D set of equations representative of the mean 2-D flow.

The 2-D Navier-Stokes equations are averaged in the $y$ direction which is orthogonal to the mean flow. The area-averaging and the Favre-averaging operators of a quantity $f$ are defined as:

$$
\bar{f}=\frac{1}{\Delta y} \int_{0}^{\Delta y} f d y \quad \widetilde{f}=\frac{1}{\Delta y \bar{\rho}} \int_{0}^{\Delta y} \rho f d y
$$

where $\Delta y$ is the height of the computational domain. The fluctuating parts of the quantity $f$ are defined as:

$$
f=\widetilde{f}+f^{\prime} \quad f=\bar{f}+f^{\prime \prime}
$$




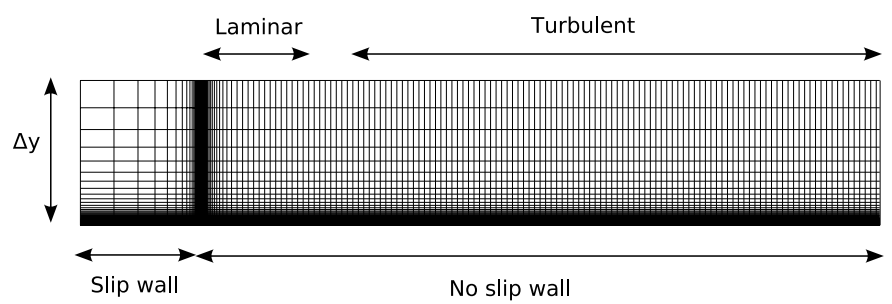

Figure 1. Flat plate definition and mesh

The rules for averaging the $\mathrm{x}$ and $\mathrm{y}$ derivatives are:

$$
\frac{\overline{\partial f}}{\partial x}=\frac{\partial \bar{f}}{\partial x} \quad \frac{\overline{\partial f}}{\partial y}=\frac{1}{\Delta y}(f(\Delta y)-f(0))=\frac{1}{\Delta y}[f]_{0}^{\Delta y}
$$

\section{Development of a boundary layer over a flat plate}

The development of a boundary layer over a flat plate is presented. The computational domain is reproduced at the figure 1. Applying the averaging operator defined above to the 2-D equations of conservation and to the flat plate problem gives the following set of equations for mass, momentum and energy balances:

$$
\begin{gathered}
\frac{\partial \bar{\rho} \widetilde{V}_{x}}{\partial x}=0 \\
\frac{\partial\left(\bar{\rho} \widetilde{V}_{x} \widetilde{V}_{x}+\bar{p}\right)}{\partial x}+\frac{\partial \overline{\rho V_{x}^{\prime} V_{x}^{\prime}}}{\partial x}=\frac{\partial \overline{\tau_{x x}}}{\partial x}+\frac{1}{\Delta y} \tau_{x y}(0) \\
\frac{\partial \bar{\rho} \widetilde{V}_{x} \widetilde{V}_{y}}{\partial x}+\frac{\partial \overline{\rho V_{x}^{\prime} V_{y}^{\prime}}}{\partial x}=\frac{\partial \overline{\tau_{x y}}}{\partial x}+\frac{1}{\Delta y} \tau_{y y}(0)-\frac{1}{\Delta y}[p]_{0}^{\Delta y} \\
\frac{\partial \bar{\rho} \widetilde{V}_{x} \widetilde{H}}{\partial x}+\frac{\partial \overline{\rho V_{x}^{\prime} H^{\prime}}}{\partial x}=\frac{\partial\left(\overline{\tau_{x x} V_{x}}+\overline{\tau_{x y} V_{y}}-\overline{q_{x}}\right)}{\partial x}
\end{gathered}
$$

where

$$
\widetilde{H}=\widetilde{h}+\frac{1}{2} \widetilde{V} \widetilde{V}+\frac{1}{2} k \quad k=\frac{1}{\bar{\rho}}\left(\overline{\rho V_{x}^{\prime} V_{x}^{\prime}}+\overline{\rho V_{y}^{\prime} V_{y}^{\prime}}\right)
$$

This system of equations is solved on a 1-D mesh. Its unknowns are the averaged conservative variables: $\bar{\rho}, \bar{\rho} \widetilde{V}_{x}, \bar{\rho} \widetilde{V}_{y}, \bar{\rho} \widetilde{H}$. Similarly to the circumferentially-averaged 3-D Navier-Stokes equations, additional terms appear in the momentum and in the energy equations. They can be cast in:

- wall stresses: $\frac{1}{\Delta y} \tau_{y y}(0), \frac{1}{\Delta y} \tau_{x y}(0)$ and $\frac{1}{\Delta y}[p]_{0}^{\Delta y}$. However these terms will be reduced to the shear stress at the wall $\tau_{x y}(0)$ since $\tau_{y y}(0)$ is negligible with respect to the shear stress $\tau_{x y}(0)$ and since the static pressure is nearly uniform in the direction perpendicular to the mean flow

- velocity-velocity stresses $\overline{\rho V_{x}^{\prime} V_{y}^{\prime}}$ and $\overline{\rho V_{x}^{\prime} V_{x}^{\prime}}$

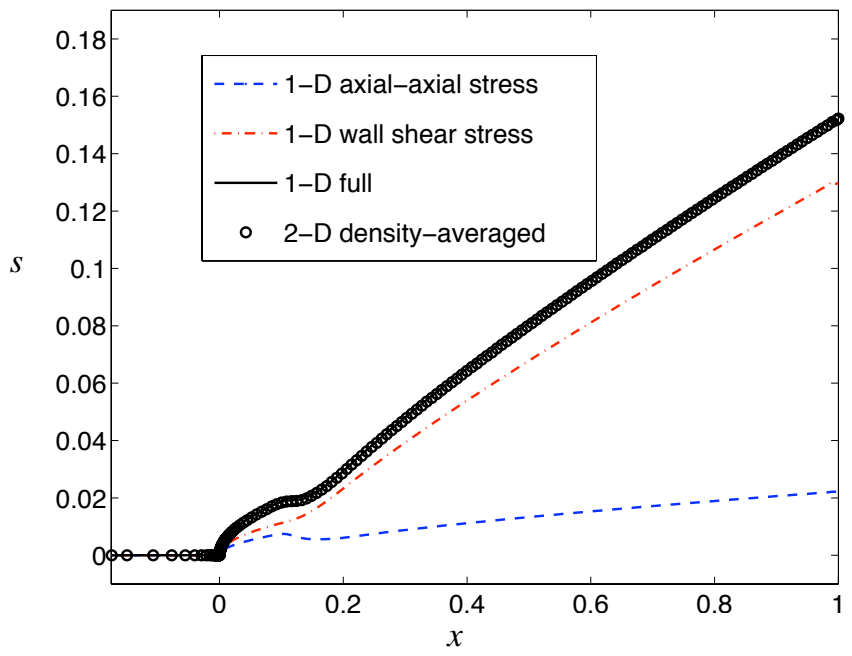

Figure 2. Impact of the additional terms on entropy along the flat plate

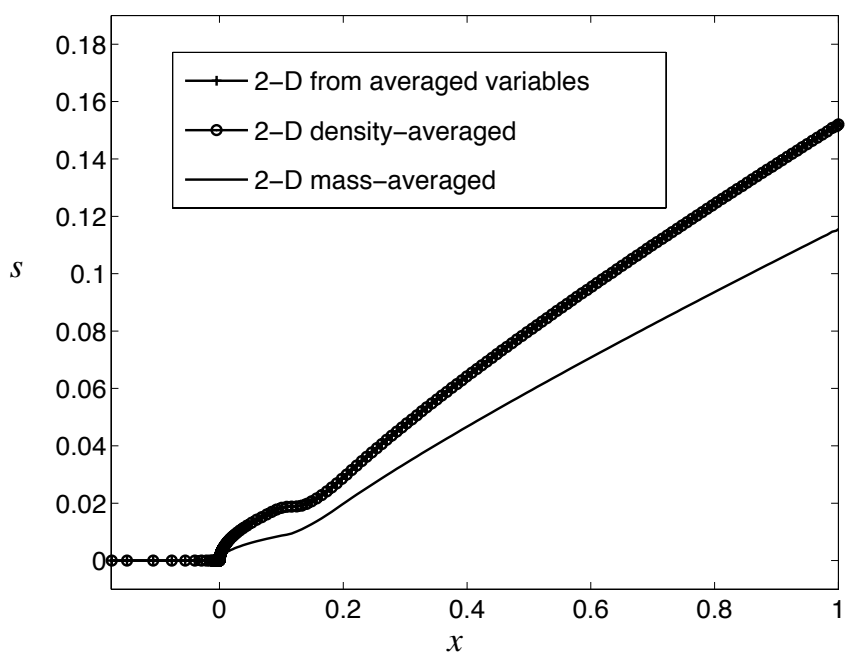

Figure 3. Mass-averaged vs density-averaged entropy

- velocity-enthalpy stress $\overline{\rho V_{x}^{\prime} H^{\prime}}$

These contributions can be computed from the 2-D flow field and included in the 1-D solver, as well as the averaged viscous stresses $\overline{\tau_{x y}}, \overline{\tau_{x x}}$ and the viscous contribution to the energy equation $\overline{\tau_{x x} V_{x}}+\overline{\tau_{x y} V_{y}}-\overline{q_{x}}$.

By switching on or off individually the terms computed from the 2-D flow field, their individual impact on the 1-D averaged flow field can be evaluated and the predominant terms can be determined. These dominant terms are the axial-axial spatial correlation $\overline{\rho V_{x}^{\prime} V_{x}^{\prime}}$ and the viscous shear stress at wall $\frac{1}{\Delta y} \tau_{x y}(0)$.

The effect of these two terms on the entropy is illustrated on the figure 2. The wall shear stress takes the greatest importance and it is the sole viscous term that is worth to be modelled in order to close the system of equations. For a separated bound- 


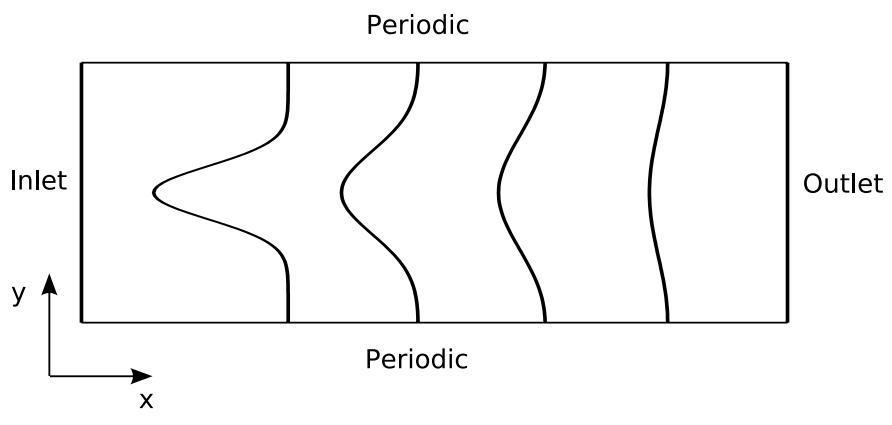

Figure 4. Wake mixing problem

ary layer, the conclusion concerning the relative importance of the axial-axial spatial stress and the viscous shear stress at wall would be inverted as the former would predominate the momentum balance. This will be illustrated on a stator corner stall in the section dedicated to the compressor testcase.

The specific entropy is an intensive quantity that defines a property per unit of mass. Therefore, a meaningful entropy average is the mass-average $\left(\bar{s}^{m}\right)$. However, the set of equations used here, as the one defined by Adamczyk, is based on a densityaverage. The entropy that is obtained is therefore a densityaveraged quantity $(\widetilde{S})$. In [6], a relation between the density timeaveraged entropy and the mass time-averaged entropy is given. Applied to the circumferential averaging, it gives the following relation between both average definitions:

$$
\bar{s}^{m}=\widetilde{s}+\frac{\widetilde{V_{x}^{\prime} s^{\prime}}}{\widetilde{V_{x}}}
$$

As shown at the figure 3 the difference between both definitions is far to be negligible.

The density-averaged entropy has been computed with the Gibbs equation directly applied to the averaged variables, although the rigorous and correct way to obtain the entropy is to apply the average operator to the Gibbs equation. It is shown in [19] that for this test-case, characterized by a low level of the kinetic energy of the fluctuation, the difference between the two approaches is negligible, as shown on figure 3 .

\section{Wake mixing}

The second test-case is a wake mixing due to the viscosity. The flow domain is a rectangle. The wake is injected as a deficit of total pressure through the left boundary and travels to the right (figure 4). The deficit of total pressure follows a cosine law and corresponds to a deficit of half of the velocity at the inlet of the domain. Periodic boundary conditions are imposed on the top and on the bottom boundaries. The mesh is uniform in both directions.

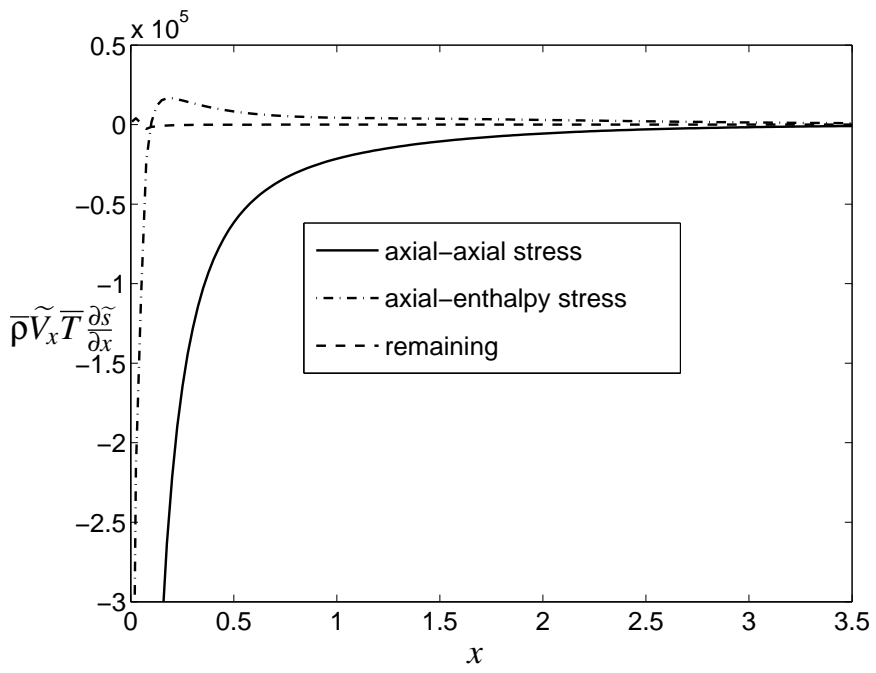

Figure 5. Contributions to entropy production in the wake

Applying the averaging procedure to the 2-D Navier-Stokes equations for this periodic wake mixing problem gives the following momentum equations:

$$
\begin{array}{r}
\frac{\partial\left(\bar{\rho} \widetilde{V}_{x} \widetilde{V}_{x}+\bar{p}\right)}{\partial x}+\frac{\partial \overline{\rho V_{x}^{\prime} V_{x}^{\prime}}}{\partial x}=\frac{\partial \overline{\tau_{x x}}}{\partial x} \\
\frac{\partial \bar{\rho} \widetilde{V}_{x} \widetilde{V}_{y}}{\partial x}+\frac{\partial \overline{\rho V_{x}^{\prime} V_{y}^{\prime}}}{\partial x}=\frac{\partial \overline{\tau_{x y}}}{\partial x}
\end{array}
$$

The mass and energy balances are identical to (11) and (14). Studying the evolution of the entropy is again the main issue. An equation for the entropy evolution is obtained by multiplying the momentum balance by the velocity field, subtracting with the energy balance and introducing the Gibbs equation. The following equation is obtained:

$$
\begin{aligned}
\bar{\rho} \widetilde{V}_{x} \bar{T} \frac{\partial \widetilde{s}}{\partial x}= & -\frac{\partial \overline{\rho V_{x}^{\prime} H^{\prime}}}{\partial x}-\bar{\rho} \widetilde{V}_{x} \frac{1}{2} \frac{\partial k}{\partial x}+\frac{\partial \overline{\rho V_{x}^{\prime} V_{x}^{\prime}}}{\partial x} \widetilde{V}_{x}+\frac{\partial \overline{\rho V_{x}^{\prime} V_{y}^{\prime}}}{\partial x} \widetilde{V}_{y}+ \\
& \frac{\partial \overline{\tau_{x x} V_{x}^{\prime}}}{\partial x}+\frac{\partial \overline{\tau_{x y} V_{y}^{\prime}}}{\partial x}+\overline{\tau_{x x}} \frac{\partial V_{x}}{\partial x}+\overline{\tau_{x y}} \frac{\partial V_{y}}{\partial x}-\frac{\partial \bar{q}_{x}}{\partial x}
\end{aligned}
$$

Compared to the equation describing the entropy production in a non-averaged flow, additional terms show up, namely the spatial stresses. The figure 5 summarizes the different contributions to the entropy production : the major contributions are those of the axial-axial stress due to $\overline{\rho V_{x}^{\prime} V_{x}^{\prime}}$ and of the axial-enthalpy stress due to $\overline{\rho V_{x}^{\prime} H^{\prime}}$. The remaining terms, including all mean viscous shear stresses, exert no influence on the 1-D evolution of entropy in a wake mixing.

The evolution of the entropy is given on the figure 6 . The density-averaged entropy exhibits a very unconventional evolution as it continuously decreases from the inlet to the outlet of 


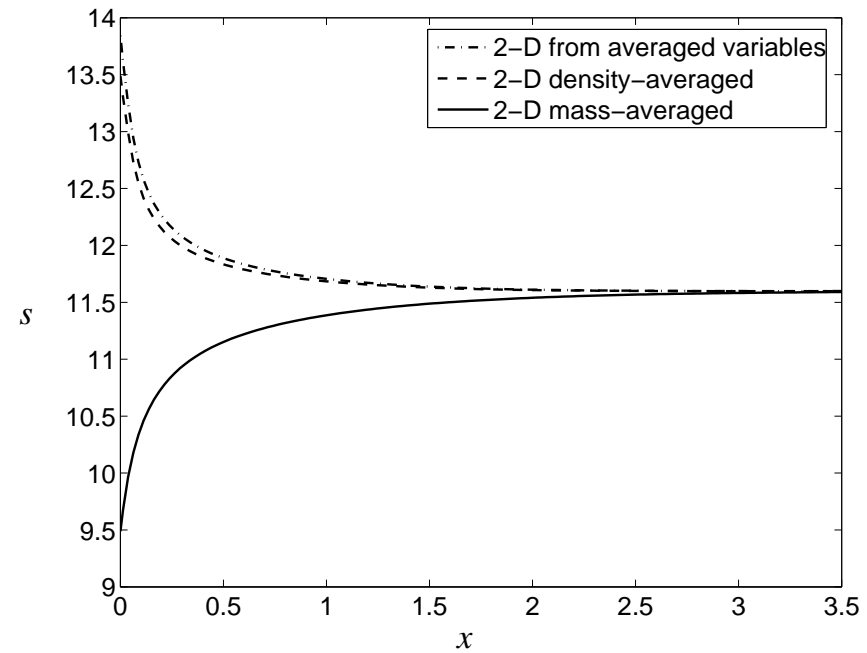

Figure 6. Entropy evolution in the wake

the domain. Looking at the equation (19) and retaining only the main contribution, i.e. $\overline{\rho V_{x}^{\prime} V_{x}^{\prime}}$, it is clear that the density-averaged entropy can only decrease in the main direction of propagation of a wake, i.e. the $x$ direction. Indeed, due to the homogenization of the wake flow, $V_{x}^{\prime}$ decreases in this direction.

For this test-case, the error made by applying the Gibbs relation directly to the averaged variables is also weak as shown on figure 6.

\section{Concluding remarks on the 1-D test-cases}

From the two test-cases presented above, the following conclusions and remarks can be made:

1. The prioritization of the different contributions to the averaged conservation equations has shown that among all viscous terms, the wall shear stress seems to be the sole viscous contribution to be important. The stresses due to the fluctuations are also important. In the case of a blade passage, some additional circumferential stresses will be generated due to the turning of the flow.

2. The error due to the application of the Gibbs equation directly to the averaged variables instead of the averaged form of the Gibbs equation seems to be weak.

3. The difference observed between the mass-averaged and the density-averaged entropy increases with the non-uniformity of the flow. Extrapolated to the circumferential-average and to a turbomachine configuration, it means that sufficiently far from the inlet and the outlet of a blade row, in the regions where the flow is circumferentially uniform (or close to), the two averages coincide (see figure 6). The global performances or radial surveys that would be obtained at the outlet of a machine with a density-average-based computation would thus be correct. Another solution would be to de- vise a mass-averaged throughflow model. Such an approach has been studied by a few authors (see [12] for example).

4. Continuing on the subject of the difference between mass and the density-averages, Bardoux [6] has also observed the particular behavior of the density-based average in the frame of 3-D steady simulations including the effect of the unsteady deterministic flow, i.e. for the time-average. The effect is however expected to be much more important in the circumferential-average problem since the kinetic energy of the circumferential fluctuations can represent a great part of the total kinetic energy. For example, in a recirculation zone, where the averaged velocity reaches zero, the fluctuations represent $30 \%$ of the maximum kinetic energy found in the complete flow field. This is in strong contrast with the turbulent kinetic energy or the deterministic kinetic energy and explains why the nature of the averaging is more critical in a throughflow problem.

\section{LOW SPEED COMPRESSOR STAGE}

The CME2 is a single stage low speed compressor. Its global performances can be summarized as follows: mass flow $11 \mathrm{~kg} / \mathrm{s}$, pressure ratio 1.14 , efficiency 0.92 , rotational speed 6300 RPM. A meridional view of the flow path and of the mesh used for the throughflow calculations is shown on the figure 7. A 3-D simulation has been performed by Gourdain [11] with the numerical code elsA developed by the ONERA. The turbulent viscosity is computed using the model of Spalart-Allmaras. Wall functions have been used. The 3-D mesh is composed of approximately one million grid points. The simulation has been run for the nominal conditions. A comparison of the results of the simulation and the experimental measurements are presented in [11]. The agreement is good.

\section{Throughflow computation with additional terms}

In the previous section it was shown for an averaged 2-D flow that the sole viscous shear stresses that play a significant role in the momentum and energy balances are the viscous shear stresses at wall, i.e the viscous blade forces. Transposed to a circumferentially-averaged 3-D flow, this means that the mean viscous shear stresses acting in the blade-to-blade surface, $\overline{\tau_{x \theta}}$ for example, can be neglected. But contrary to the blade walls, the annulus endwalls are present in the throughflow model. The contribution of the viscous stresses in these regions is expected to be important and is therefore not neglected.

The inviscid and viscous blade forces and the circumferential stresses have been extracted from the 3-D simulations and injected in the Navier-Stokes throughflow model. The sole viscous term extracted from the 3-D simulation is the viscous blade force. All other viscous terms are neglected or modelled by the 2-D axisymmetric Navier-Stokes viscous terms coupled to the 


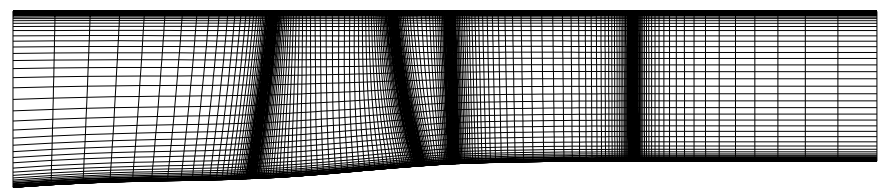

Figure 7. Meridional mesh of the CME2 compressor

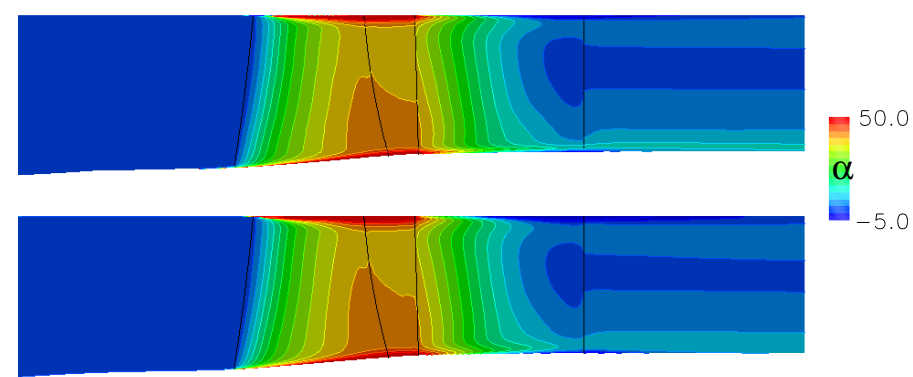

Figure 8. Absolute flow angle from 3-D averaged solution (top) and throughflow solution (bottom)

Baldwin-Lomax turbulence model. The resulting model is refered to as the high-order throughflow model.

A typical mesh used for such computations is presented at the figure 7. The mesh is clustered around the leading and trailing edges to capture the strong gradients typical of these regions.

A qualitative overview of the obtained solution is presented on the figure 8 which compares the absolute flow angle for the throughflow solution and the averaged 3-D solution. The agreement is impressive. All the flow features are reproduced, such as the recirculation in the tip gap region or the stator corner stall.

Quantitative investigations can be performed by inspecting radial surveys at inlet, outlet but also inside each blade rows as shown on figures 9 for the rotor and 10 for the stator. The flow field inside the rotor is really well reproduced by the extracted 3 -D terms and the viscous endwall model. For the stator row, the agreement is still good but some discrepancies occur in the corner stall region.

The discrepancies can be attributed to either numerical or modelling errors. The numerical errors can be due to the interpolation operations needed to compute the forces and the stresses from the 3-D flow field, to the discretization of the stresses themselves in the throughflow model (by a Green-Gauss scheme) and/or to the discretization errors in the 3-D solution itself.

The modelling errors are related to the assumptions used to build the throughflow model : only the blade-to-blade viscous blade force contribution is included, the averaged viscous stresses are missing. Secondly, the endwall losses are modelled by the axisymmetric Navier-Stokes equations which is a simplification compared to the 3-D viscous terms circumferentially averaged. Finally, the turbulence model used in the throughflow (the Baldwin-Lomax model) is different from the one used in the
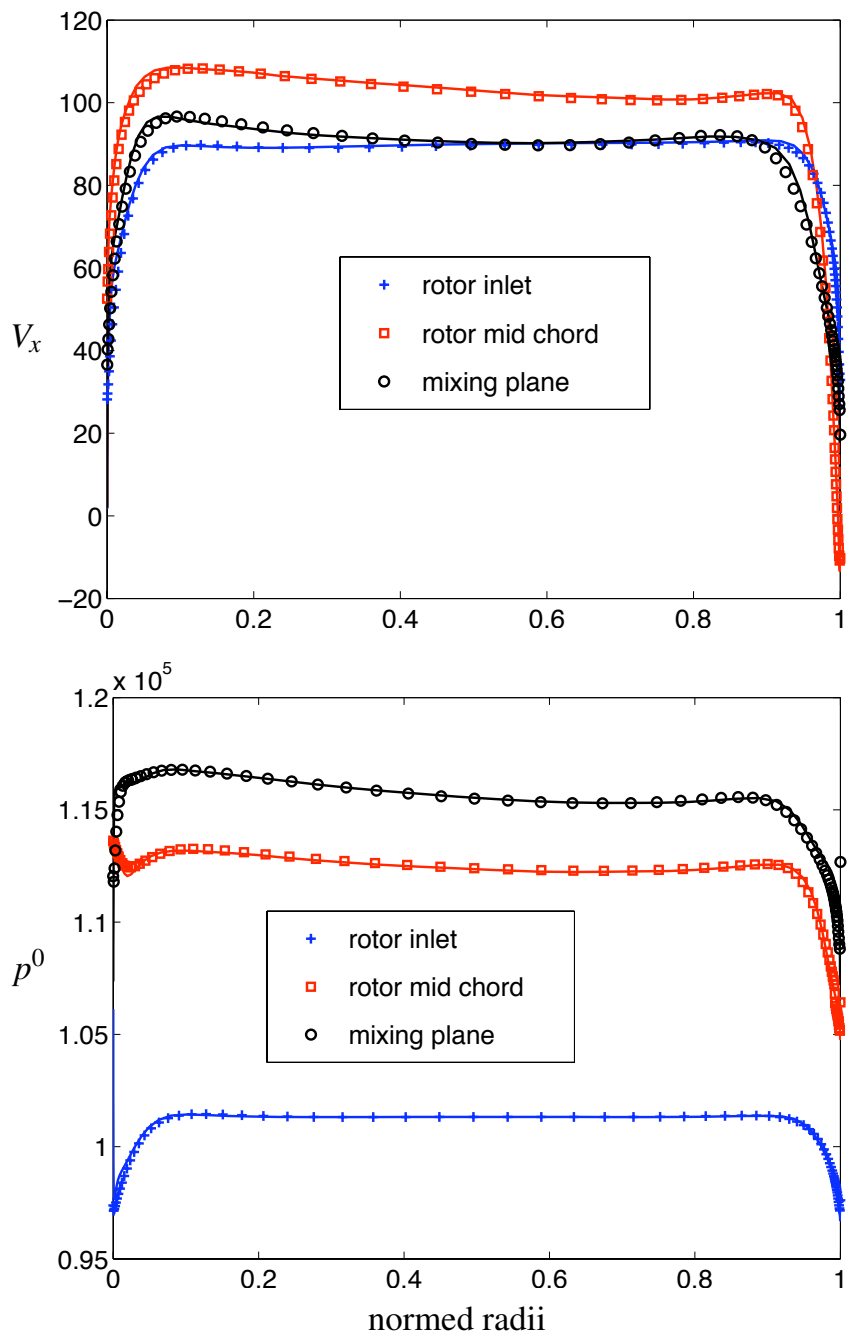

Figure 9. Comparison between the throughflow solution (plain lines) and the 3-D solution circumferentially averaged (symbols) at 3 locations inside the rotor domain

3-D computation (the Spalart-Allmaras model coupled to wall functions).

In the present case, the main discrepancy has been attributed to the 2-D Baldwin-Lomax turbulence model. A computation has been run with a fixed turbulent viscosity extracted from the 3-D computation circumferentially averaged : the total pressure peaks in the boundary layer have disappeared along both walls. In these regions, the new total pressure distribution fits very well the averaged 3-D one. In conclusion, the agreement between the throughflow solution and the 3-D averaged solution is considered to be very good. The main improvement lies in introducing a more elaborated turbulence model. A standard 2-D model in the hub-to-tip direction as for channel flows should be sufficient. 

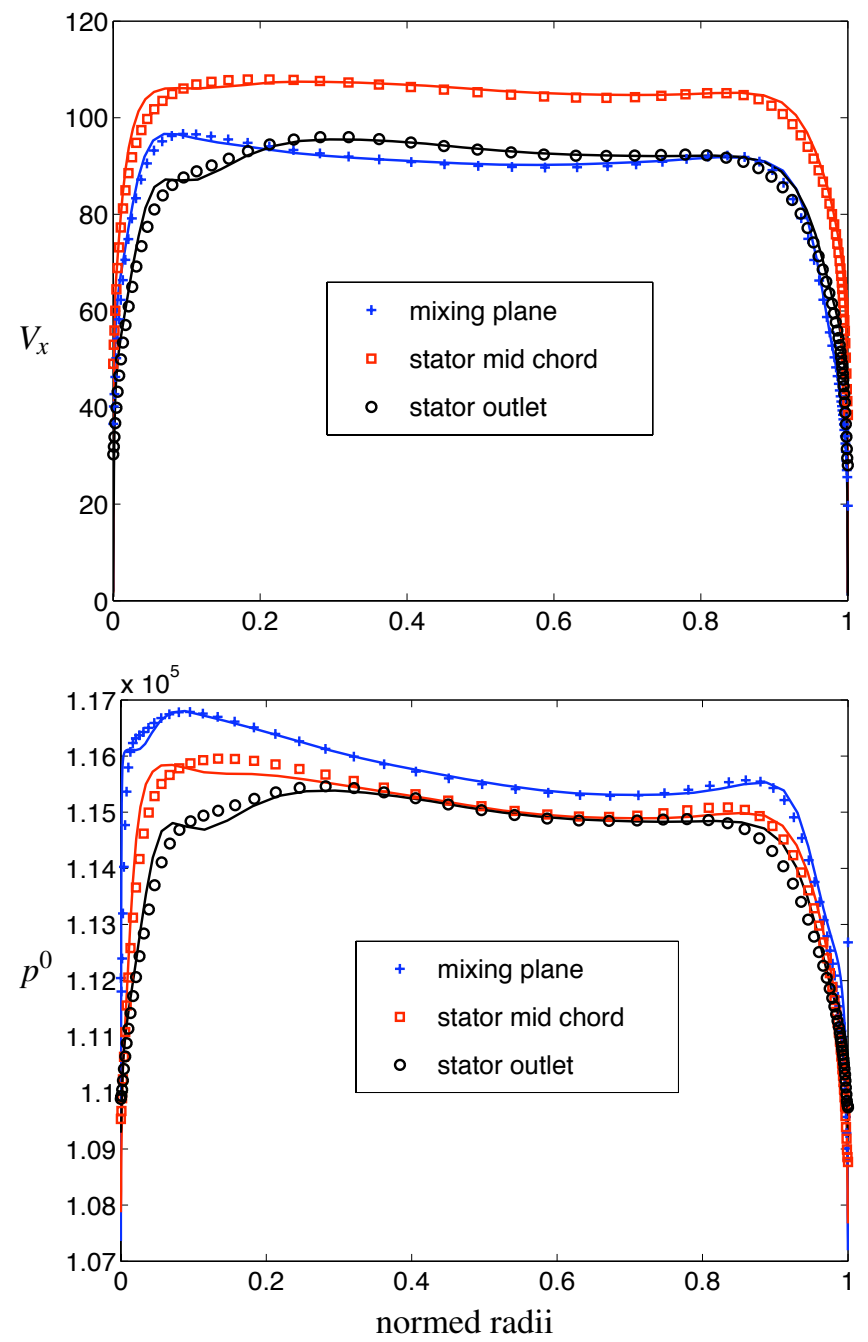

Figure 10. Comparison between the throughflow solution (plain lines) and the 3-D solution circumferentially averaged (symbols) at 3 locations inside the stator domain

\section{Hierarchy of the additional terms}

The relative importance of the different contributions taken from the 3-D simulation, i.e. the blade forces and the circumferential stresses, on the momentum and energy equations will be now evaluated. The figure 11 compares the entropy evolution for four computations:

1. a computation with the inviscid blade force only $\left(f_{b}\right)$,

2. a computation with both the inviscid and the viscous blade forces $\left(f_{b}+f_{v}\right)$,

3. a computation with the inviscid blade force and the circumferential stresses ( $f_{b}$ and stresses),

4. a full computation containing all contributions $\left(f_{b}, f_{v}\right.$ and stresses).
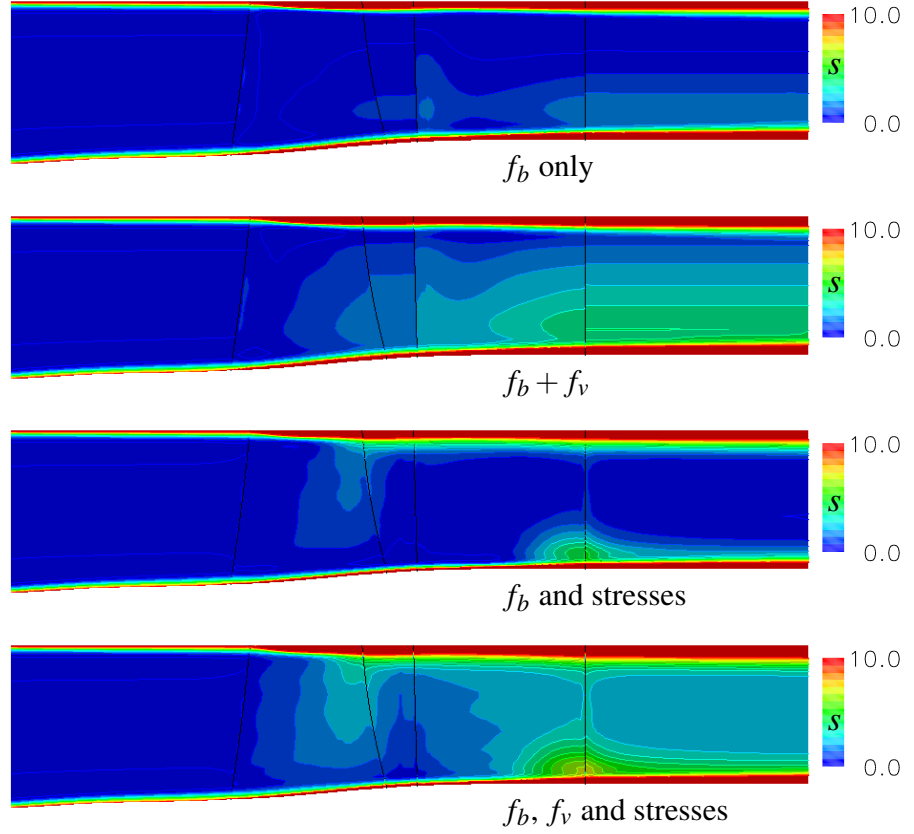

Figure 11. Impact of the blade forces and of the circumferential stresses on the entropy field

The simulation including only the inviscid blade force shows some entropy generation in the core flow region, where the annulus endwall flows have no influence. This is due to the unbalance of the momentum and energy equations. A similar result would have been obtained with a 3-D Euler simulation from which inviscid blade forces and circumferential stresses are extracted and injected in the throughflow model. An isentropic flow would be obtained with the throughflow only if all contributions are introduced in the conservation equations. Removing the stresses leads to an unbalance of the equations and to an unphysical entropy evolution.

The entropy shows also a strange behaviour close to the trailing edge for the computations including the circumferential stresses: an increase-decrease along a streamline in the core flow. The explanation for that phenomenon is the same as the one used for the wake mixing test-case presented above: it is due to the density-based average nature of the solved equations.

Both the viscous blade force and the circumferential stresses participate to the generation of entropy: the viscous blade force is more or less responsible for the level of entropy while the circumferential stresses shapes its distribution, such as in the corner stall region. This is confirmed by the inspection of the radial distribution of the entropy at the outlet of the stator (figure 12).

It is possible to gain more insight on the role of the different forces and stresses by analyzing individually their effects. These relative contributions are given at the figure 13 for the axial momentum balance. The complete analysis can be found in [19] for 


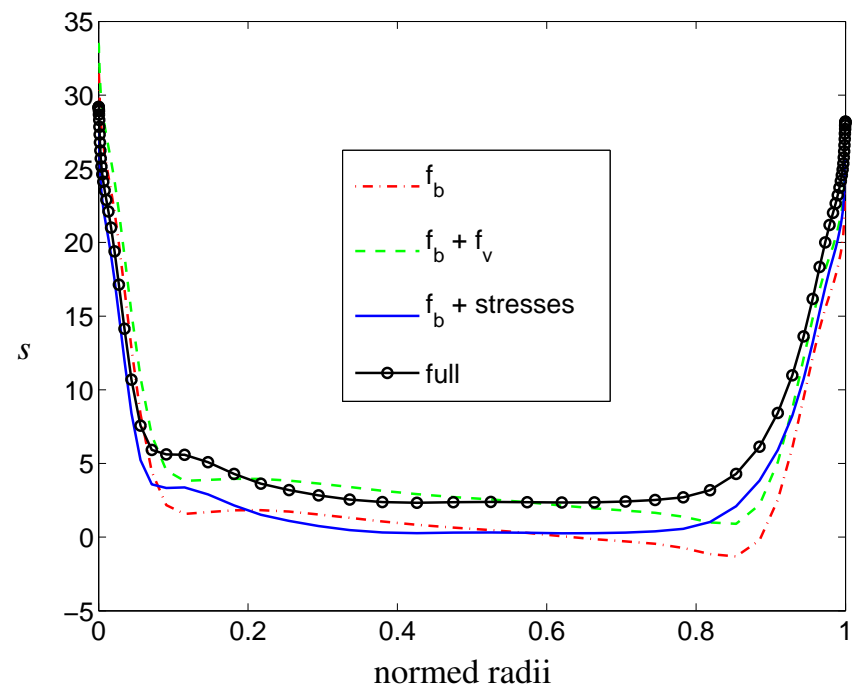

Figure 12. Impact of the blade forces and of the circumferential stresses on the radial distribution of entropy
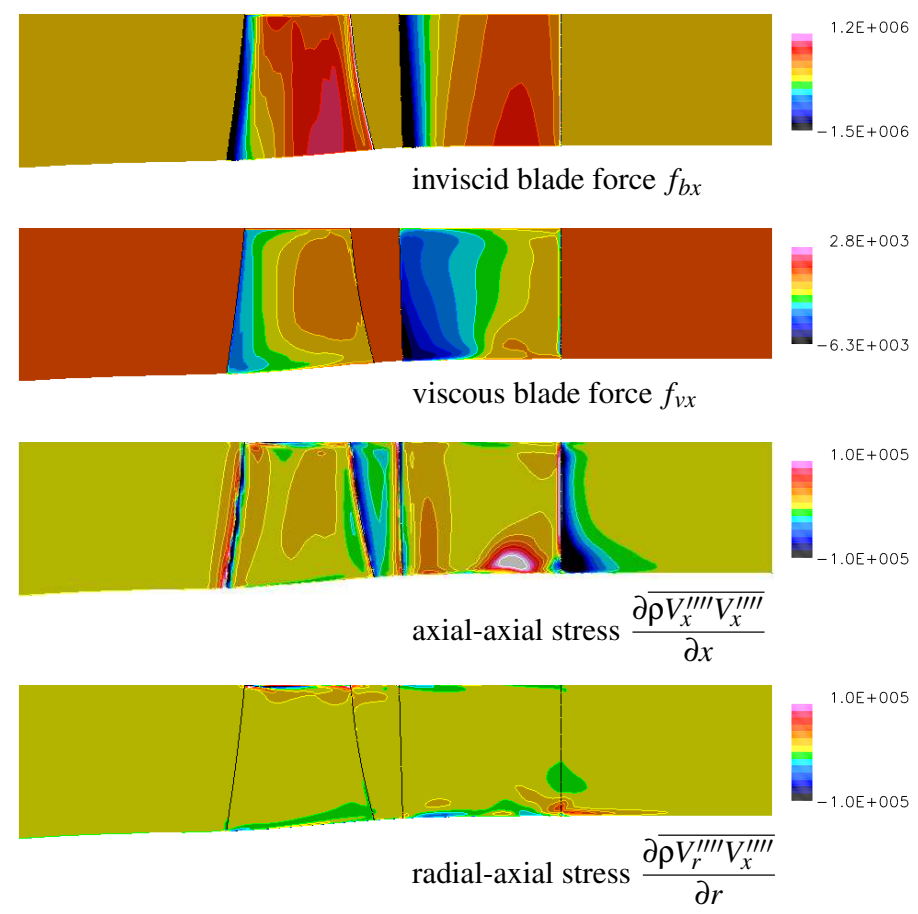

Figure 13. Contributions to the axial momentum balance

each balance equation separately.

The inviscid blade force clearly dominates the momentum balance. Next come the circumferential stresses and finally the viscous blade forces. Considering the mean level of the latter, one could conclude that it is not worth to include them in a throughflow simulation. This is misleading as another crite- rion must be taken into account, i.e. the direction of the force, which is important for the loss generation mechanism. This can be shown by the distributed loss model theory (see [13] for example). For the present test-case, neglecting the viscous blade force leads to a strong overestimation of the isentropic efficiency: 0.947 to be compared to 0.894 for the full throughflow simulation and to 0.891 for the 3-D one. This result confirms the conclusion devised from the 1-D test-cases.

This conclusion is however in contradiction with results found in the literature on the deterministic stresses closure. One way to address the deterministic stresses closure is to consider the Adamczyk assumption, i.e. neglecting the purely temporal part of the deterministic stresses and retaining only the spatial part [3]. Some approaches based on this assumption compute explicitly the terms required for the closure and neglect the viscous terms [15], [6]. Bardoux [6] has presented some results obtained on the same CME2 compressor that is studied here. He showed a comparison between the 3-D flow averaged in the stator and the same flow with the stator modelled by a "ghost row', consisting of an inviscid blade force and the circumferential stresses. He found some differences between the two computations. Following the analysis presented in this work, the discrepancy observed by Bardoux can be attributed to the absence of the viscous blade force in his stator model. Indeed, the discrepancy observed by Bardoux is the same as the one observed between the full simulation and the $f_{b}+$ stress one on the figure 12 .

\section{Classical versus high-order throughflow}

To end the analysis of the CME2 compressor, a comparison is performed between the high-order throughflow and a more classical throughflow. The latter is based on the axisymmetric Navier-Stokes equations and on empirical correlations that allow to compute the flow angle (through the deviation) and the losses (through profile loss and 3-D loss coefficients). The classical throughflow is based on mass-averaged quantities, while the high-order throughflow is explicitly based on a density-average approach. Therefore the comparison between the throughflow computations has been performed downstream of the stator, not too close to the trailing edge to avoid the difference between the density and the mass averages but not too far, to avoid a completely mixed flow. Two classical throughflow computations are presented : a computation with the Baldwin-Lomax turbulent model for the endwall flows (TF BL) and a computation with a radial mixing model (TF radial mixing). Details on these two models are given in [17].

The figure 14 shows the axial velocity. The global agreement between the classical throughflow and the 3-D results is rather good. In the hub region, the problem arising from the Baldwin-Lomax closure is visible on about $5 \%$ of the span for both the high-order throughflow and the classical throughflow without radial mixing model. For the high-order throughflow, 


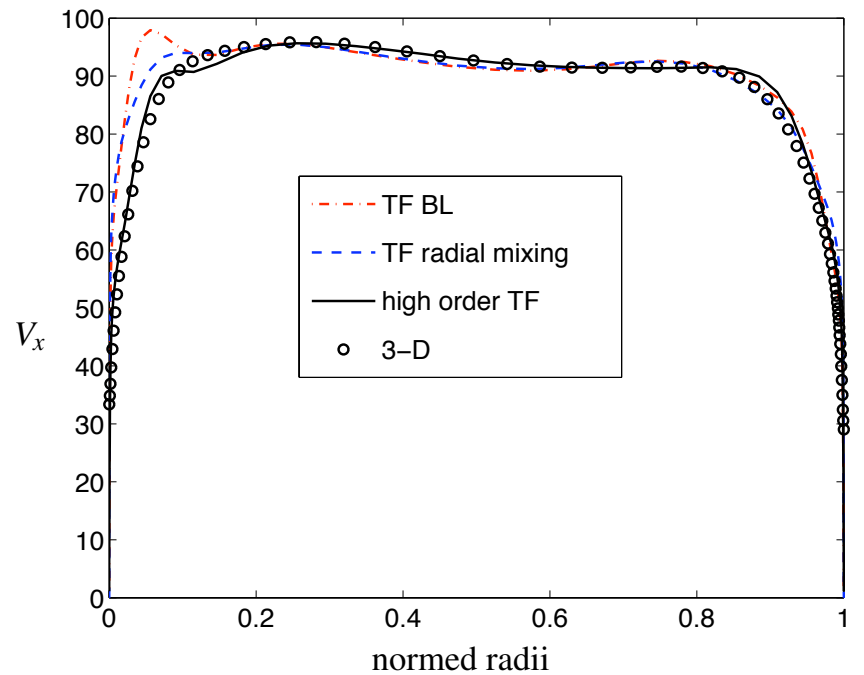

Figure 14. Classical vs high-order throughflows : axial velocity

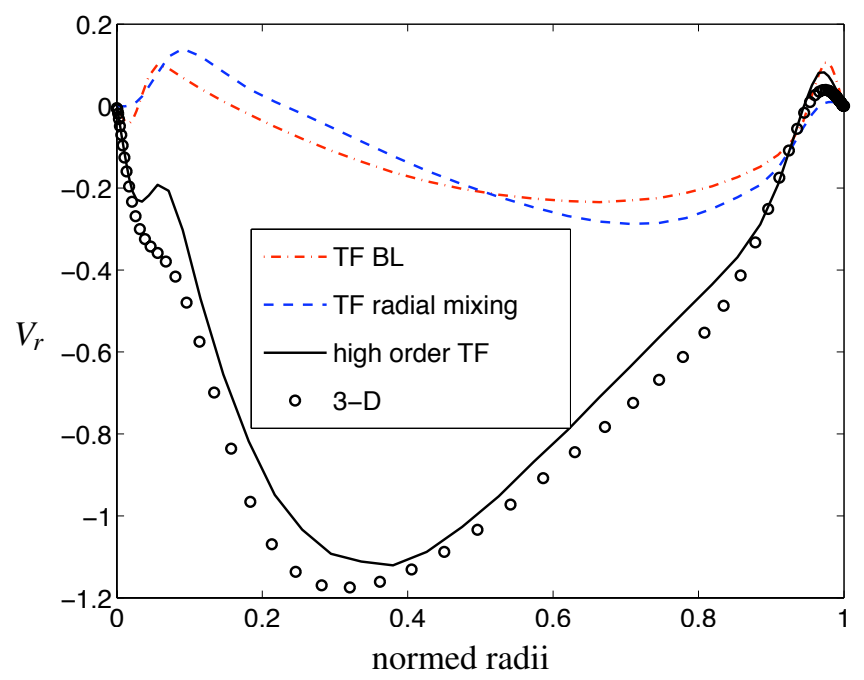

Figure 15. Classical vs high-order throughflows : radial velocity

the problem is very weak. The classical throughflow with radial mixing model over-predicts the axial velocity close to the hub wall which results in an over-prediction of the total pressure (not shown here) in that region.

From the figure 15, it is clear that the (weak) radial velocity at the outlet of the compressor can only be predicted by the circumferential stresses. The absolute flow angle is given on the figure 16. The empirical correlations used here for the classical throughflow computations mispredict the mean level of the deviation angle at the outlet of the compressor stage by 2-3 degrees. The 3-D loss correlations of Roberts [16] used in the classical throughflow, while providing the correct level of underturning,

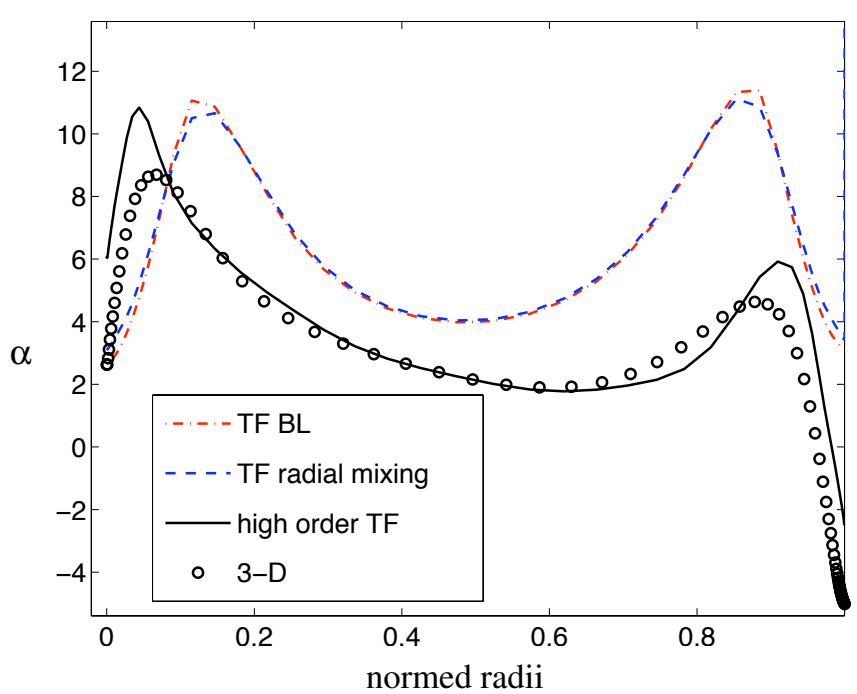

Figure 16. Classical vs high-order throughflows : flow angle

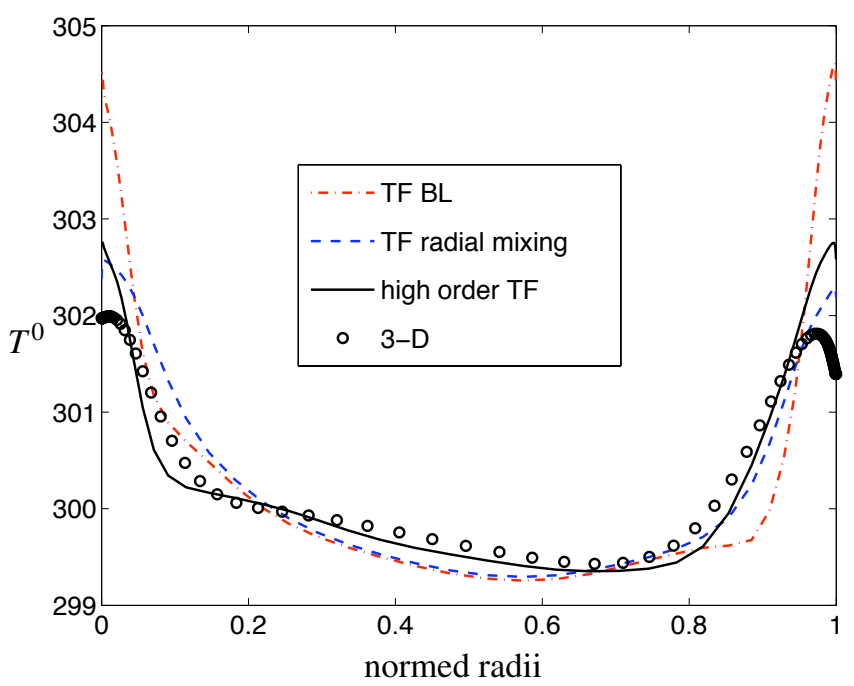

Figure 17. Classical vs high-order throughflows : total temperature

obviously fail to locate the passage vortex at the hub.

The figure 17, giving the total temperature, highlights the radial mixing phenomenon, which is relatively weak in this machine, but nevertheless present. The Navier-Stokes throughflow computation without radial mixing model over-predicts the temperature at the walls. Both the circumferential stresses and the radial mixing model in the classical throughflow computations succeed in predicting the mixing phenomenon.

Another impressive feature of the high-order throughflow model is its capability to capture the pre-rotation of the flow approaching a blade row, as illustrated by the figure 18. Indeed, due to the pure axisymmetric nature of its momentum equations, 


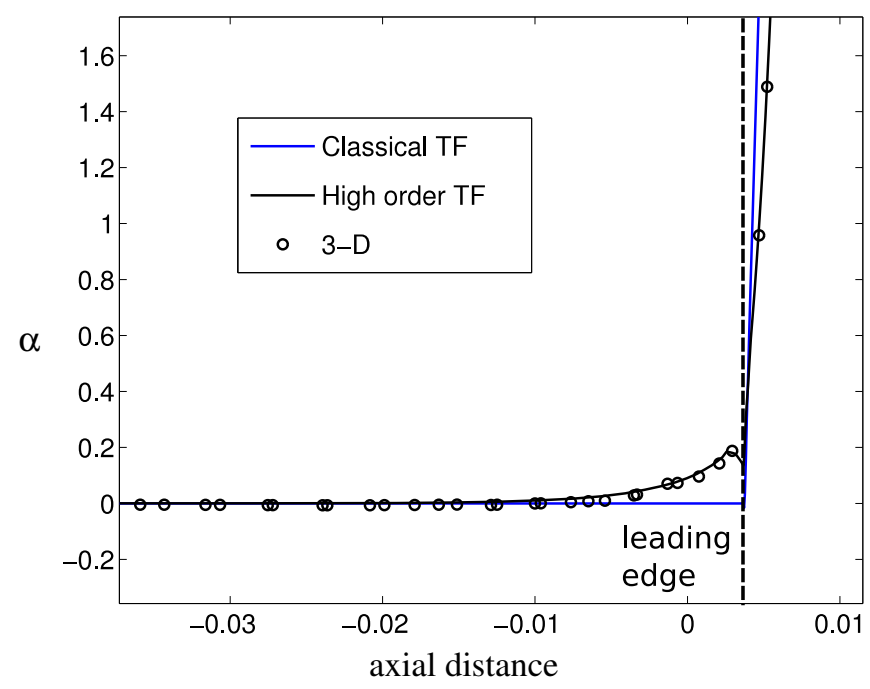

Figure 18. Pre-rotation in the leading edge region of the rotor

a classical throughflow is unable to propagate the effect of the blade force upstream of the corresponding blade row. This leads to an unphysical incidence of the incoming flow, whose angle is different from that imposed in the leading edge region by the blade model. This non adaptation may result in a strong (unphysical) entropy jump. To get rid of this undesirable artifact an adaptation of the leading edge geometry is necessary in order to smooth the transition from the axisymmetric flow direction to the imposed material direction. The figure 18 clearly shows that, due to the implementation of the circumferential stresses in the high-order throughflow model, the flow is turned upstream of the blade row, following the physics of the (averaged) 3-D simulation.

\section{TRANSONIC TURBINE STAGE}

The transonic single stage turbine VEGA2 is representative of the first stage of a high pressure turbine. Its global performances can be summarized as follows: mass flow $3.11 \mathrm{~kg} / \mathrm{s}$, pressure ratio 0.31 , efficiency 0.93 , rotational speed 13000 RPM.

Both steady and unsteady numerical simulations have been run by Bardoux et al. [7], [8] on the same multi-block structured mesh, with the flow solver CANARI-COMET developed by the ONERA. The number of grid points is approximately two millions. The simulations were run without tip clearance and with an algebraic turbulence model. A complete analysis of the simulations is available in [7], [8]. The main particularity of this testcase is recalled hereafter.

This testcase presents an important difference between the unsteady time-averaged solution and the steady one. This difference is mainly due to the radial migration of the stator wake. Indeed the static pressure is approximately constant across the

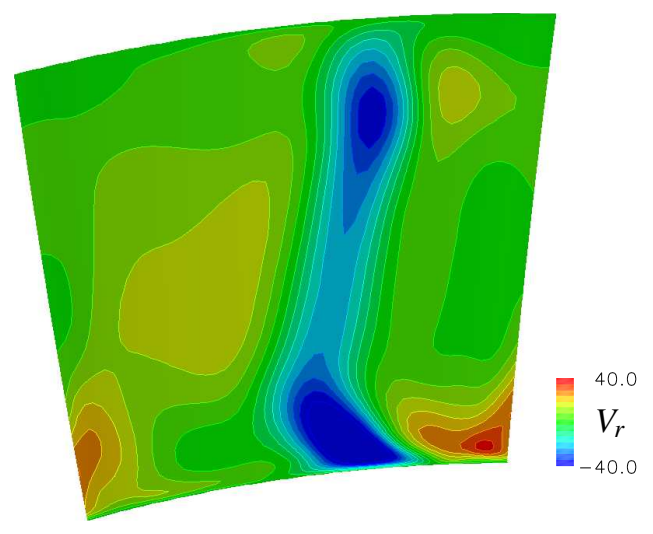

Figure 19. Time-averaged radial velocity downstream of the stator

wake for a given spanwise position. The same radial pressure gradient is therefore applied on the core flow and on the wake flow. In the core flow, the centrifugal force generated by the circumferential velocity balances the radial pressure gradient. This is not the case in the wake, resulting in its radial migration toward the hub. This is illustrated on the figure 19 where the migration of the wake flow is highlighted by the negative radial velocity. The radial migration of the stator wake plus the stator passage vortex are responsible for an accumulation of low energy fluid at the wake foot. This circumferential non-uniformity results in a strong time variation of the inlet condition of the rotor close to the hub. As a consequence, the rotor passage vortex has a pulsating character. The time-averaged representation of the passage vortex therefore appears as spread compared to the passage vortex obtained by the steady simulation obtained with a mixingplane.

\section{Throughflow computation with additional terms}

The deterministic stresses, the circumferential stresses and the blade forces have been extracted from the unsteady database and injected into the throughflow solver. The figure 20 compares the averaged 3-D unsteady simulation and the throughflow solution. The global agreement is not as good as for the CME2 testcase. The zone of high axial velocity at the stator wake foot is more extended in the throughflow calculation than in the 3-D one. The developments of the vortex, at hub and at shroud of the rotor blade, are also not correctly predicted. The global agreement is however thought to be very good.

The main discrepancy occurs close to the stator trailing edge. The losses are underestimated at the outlet of the stator as shown by the radial distributions of entropy on the figure 21 . A throughflow computation with two times more grid points in the axial direction has been performed. No noticeable differences have been observed meaning that the discretization errors are low. A possible explanation for this discrepancy is that the blade-to-blade viscous shear stresses have been neglected in the 


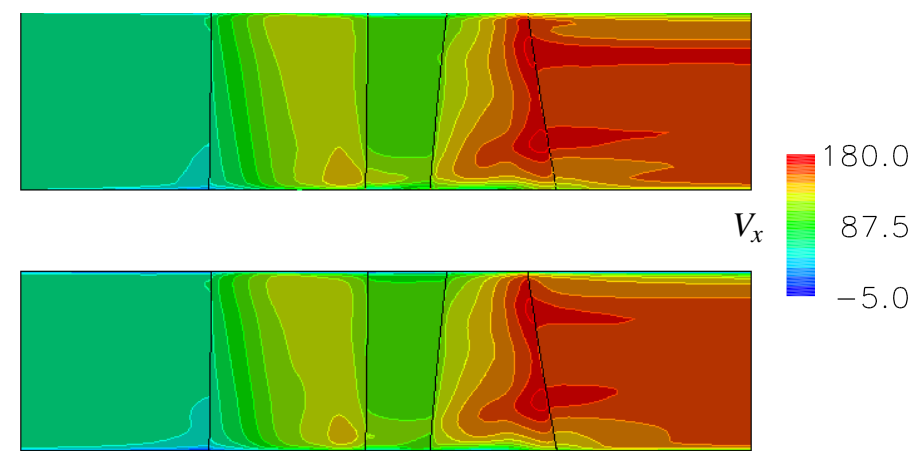

Figure 20. Axial velocity from 3-D averaged solution (top) and throughflow solution (bottom)
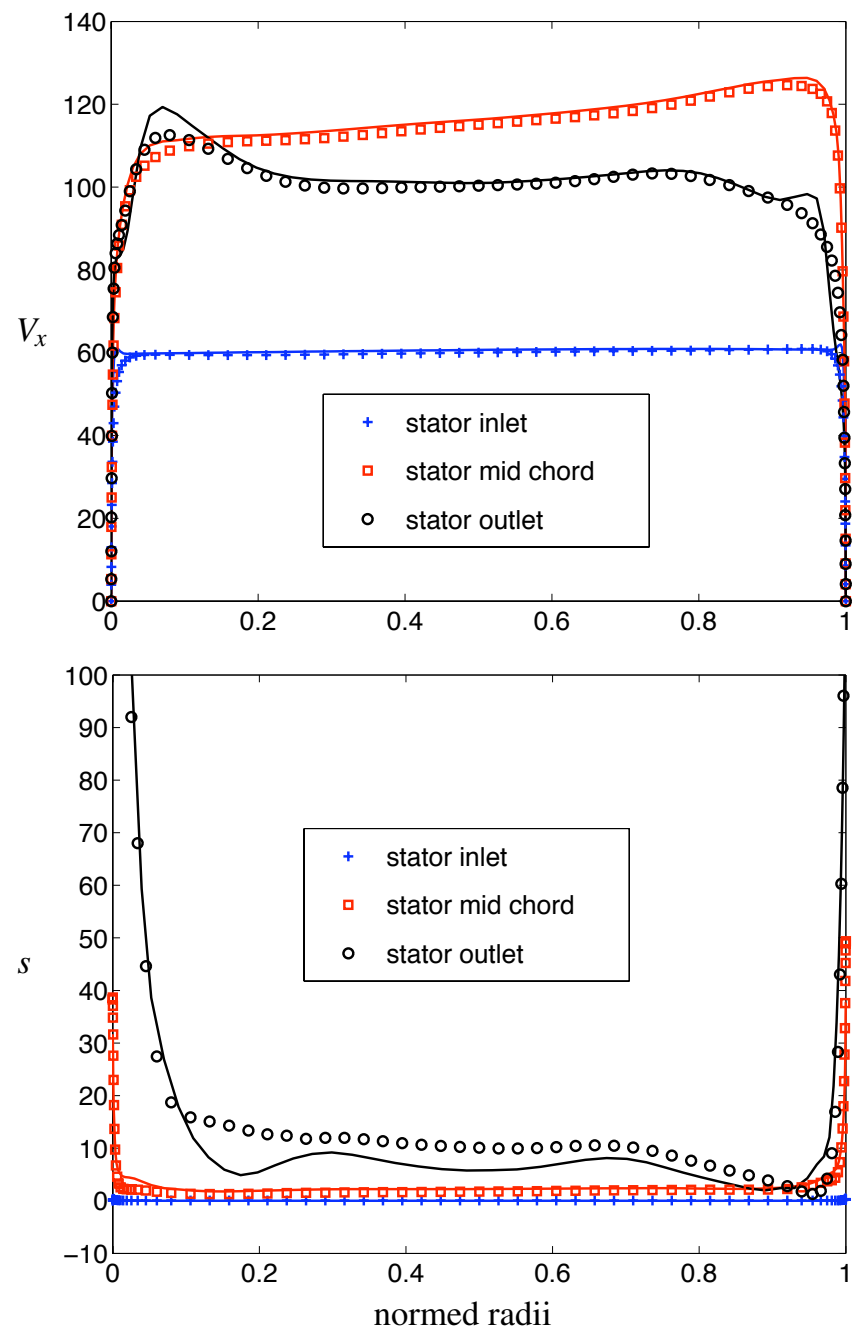

Figure 21. Comparison between the throughflow solution (plain lines) and the averaged unsteady 3-D solution (symbols) at 3 locations inside the stator domain throughflow. The conclusions concerning the impact of these stresses that were elaborated on the flat plate testcase and that have hold for the CME2 testcase could be invalid here, for the aft-part of this high-deflection stator.

The unavailability of the complete 3-D viscous stresses does not allow to go further on that subject. However, it should be noted that the discrepancy mainly affects the entropy and the other loss-related quantities, other quantities such as the axial velocity are better predicted as shown on the figure 21 .

Finally, the global performances are well predicted. The throughflow computed mass flow is equal to $3.09 \mathrm{~kg} / \mathrm{s}$ for 3.11 $\mathrm{kg} / \mathrm{s}$ in the 3-D unsteady simulation, the pressure ratio is 0.310 in the throughflow computation for 0.311 in the 3-D one and the efficiency is equal to 0.931 in the throughflow computation for 0.934 in the 3-D one.

\section{Relative importance of the deterministic and circum- ferential stresses}

Bardoux et al. $[7,8]$ showed that including the deterministic stresses in the 3-D simulation of the VEGA2 rotor allows to capture the mean effect of the unsteadiness on the mean steady flow. They also showed that the purely temporal part of the deterministic stresses cannot be neglected, contrary to the assumptions of the closure proposed by Adamczyk [3]. The study of Bardoux et al. was focused on 3-D simulations and deterministic stresses. The question of the importance of the deterministic stresses must now be addressed at the throughflow level.

As described in the model of Adamczyk, for a single shaft turbomachine, two steady flow fields exist, one per reference frame. The circumferential stresses are relative to a steady flow field in a given reference frame. In other words, the circumferential stresses field is discontinuous at the stator-rotor interface. This discontinuity is mainly present in the regions of high unsteadiness, and disappears when the deterministic stresses are superimposed to the circumferential stresses. Even though the deterministic stress are necessary in order to obtain a physically correct averaged solution, it is not clear whether neglecting them would be an important source of error. To this end three throughflow simulations are compared on the figure 22: one with the blade forces only $\left(f_{b}\right)$, one with the blade forces and the circumferential stresses $\left(f_{b}+\right.$ circ. stress $)$, and one including the blade forces, the circumferential and the deterministic stresses $\left(f_{b}+\right.$ circ. stress + det. stress $)$. The circumferential stresses show a greater influence than the deterministic stresses. They are responsible for the trace of the passage vortex in the pressure distribution. The deterministic stresses have not a predominant impact on the resulting flow field to the exception of the hub region where the unsteadiness is the strongest.

To further evaluate the error that would be brought by neglecting the deterministic stresses, three results are compared on the figure 23: those obtained without the deterministic stresses 


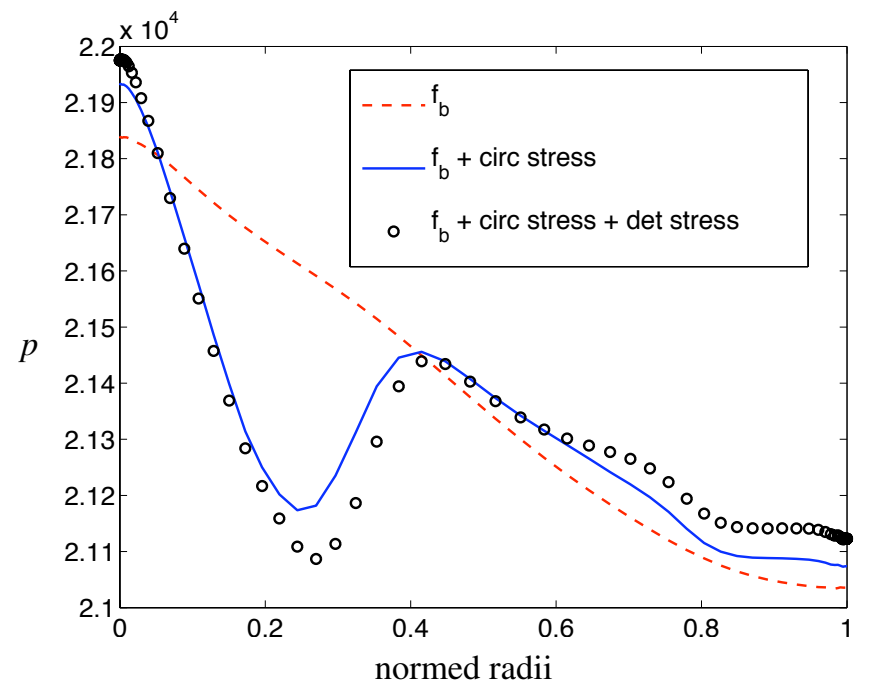

Figure 22. Comparison of the throughflow simulations with and without deterministic stresses (rotor outlet)

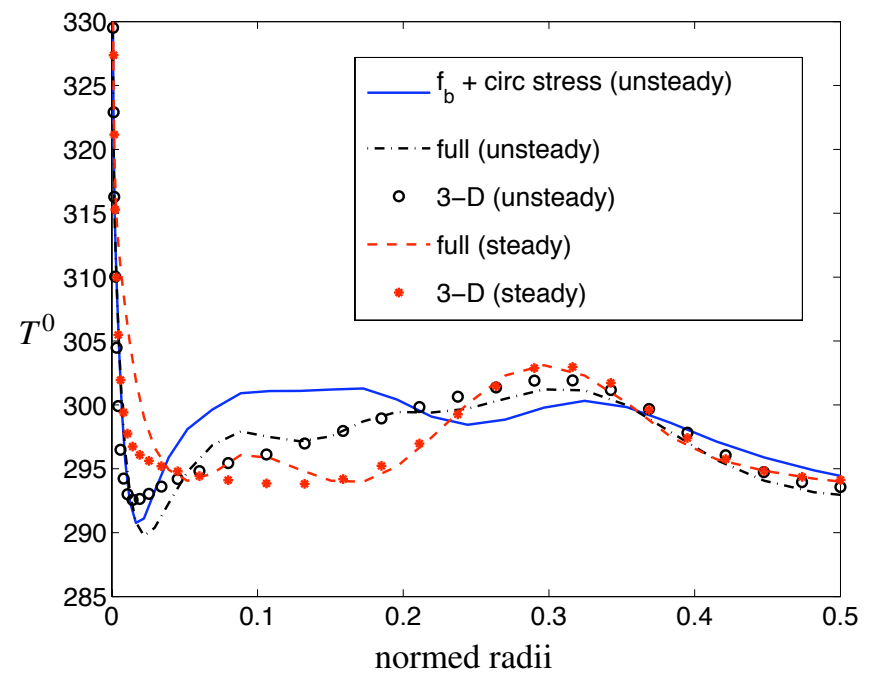

Figure 23. Comparison of the throughflow simulations with and without deterministic stresses (rotor outlet)

$\left(f_{b}+\right.$ circ stress (unsteady)), those obtained with the deterministic and the circumferential stresses extracted from the 3-D unsteady simulation (full (unsteady)) and obtained with the circumferential stresses extracted from a 3-D steady mixing-plane simulation (full (steady)). The reference 3-D unsteady simulation and the 3-D steady one are also plotted on the figure with symbols. It is obvious from this comparison that the simulation without the deterministic stresses is not accurate, as it is as far from the reference unsteady solution as the steady one is. Particularly, the radial mixing phenomenon brought by the unsteadiness is mispredicted.

\section{CONCLUSIONS}

The main motivation of this contribution was to propose a way to reduce the level of empiricism embedded in the throughflow models with the help of the high-order throughflow model.

The potential of this model to capture all flow features has been demonstrated. Concerning the closure of the (steady) problem, it has been shown that the following terms are necessary to correctly reproduce the flow physics: the inviscid blade force, the circumferential stresses, the viscous blade force and a 2-D viscous model for the endwall flows. The most important contribution is obviously the inviscid blade force. The relative importance of the circumferential stresses increases with the loading. When unsteadiness effects are important, the deterministic stresses should also be included even though they are not preponderant compared to the circumferential ones.

The improvement brought by the high-order throughflow model over a more conventional throughflow model has been shown for the CME2 testcase. It consists in a more accurate capture of the boundary layer flow and of the radial velocity field. The loss coefficient and deviation are also accurately captured.

The features of the high-order throughflow system of equations have also been highlighted in this contribution. The main particularity is probably the evolution of the entropy due to the Favre averaging chosen to derive the equations. This behavior will be difficult to modify unless another system of equations is devised, based on mass-averaged properties.

Nothing has been done on the modelling of the different terms needed to close the equations. Some thoughts on how to address this closure are given hereafter.

For the inviscid blade forces, we believe that two solutions are available:

- to use fast 3-D models to compute the pressure distribution on the blade walls, such as potential methods (singularity methods) or Euler ones.

- to use blade force models found in classical Euler and Navier-Stokes finite volume throughflows [5, 17]. This method is valid for both analysis calculation where the geometry is known and design calculation where a blade loading is prescribed. With these models, a physically correct isentropic flow is obtained for a shock free inviscid computation. However, the addition of the circumferential stresses modify this isentropic result. The blade force model (more specifically the orthogonality condition it imposes between the blade force and the mean flow) should be adaptated to account for the presence of the circumferential stresses.

For the circumferential stresses, adapting techniques inspired from the deterministic stresses closure seems a natural way, among them are transport equations for the circumferential stresses or the non linear harmonic modelling. A project studying the feasibility of a circumferential closure by a method inspired 
from the non linear harmonic method is currently undertaken by Thomas [23]. Some modifications of the original method will be necessary concerning the function chosen to represent the fluctuations.

Finally, for the viscous blade force, a simple model giving the skin friction on the blade walls could be obtained from typical relations used for wall function formulations. Considering that the circumferential stresses closure will be able to bring some information on the evolution of the velocity in the direction orthogonal to the blade walls, it will be possible to compute the viscous blade force.

\section{ACKNOWLEDGEMENTS}

The authors would like to express their deep gratitude to Francis Leboeuf (Ecole Centrale de Lyon) for his valuable advises, and to Stéphane Burguburu (ONERA) who provided the 3-D testcases CME2 and VEGA2.

\section{REFERENCES}

[1] J.J. Adamczyk. Model Equation for Simulating Flows in Multistage Turbomachinery. NASA-TM-86869, 1984.

[2] J.J. Adamczyk. Aerodynamic Analysis of Multistage Turbomachinery Flows in Support of Aerodynamic Design. ASME Paper 99-GT-80, 1999.

[3] J.J. Adamczyk, R.A. Mulac and M.L. Celestina. A Model for Closing the Inviscid Form of the Average-Passage Equation System. Transactions of the ASME Journal of Turbomachinery, 108, 180186, 1986.

[4] M.S. Attia. Semiviscous Method for Compressor Performance Prediction. Journal of Propulsion and Power, 21(5), 792796, 2005.

[5] S. Baralon. On multistage Analysis of Transonic Compressors : From axisymmetric Throughflow Time Marching to Unsteady Three Dimensional Methods. Ph.D. thesis, Chalmers University of Technology, 2000.

[6] F. Bardoux. Modélisation des interactions instationnaires rotor-stator en turbomachine multi-étages. Ph.D. thesis, Ecole centrale de Lyon, 2000.

[7] F. Bardoux, F. Leboeuf, C. Dano and C. Toussaint. Characterization of Deterministic Correlations for a Turbine Stage. Part 1 : Time Averaged Flow Analysis. ASME Paper 99GT-100, 1999.

[8] F. Bardoux, F. Leboeuf, C. Dano and C. Toussaint. Characterization of Deterministic Correlations for a Turbine Stage. Part 2 : Unsteady Flow Analysis. ASME Paper 99-GT-100, 1999.

[9] N.A. Cumpsty. Compressor Aerodynamics. Longman Scientific and Technical, 1989.
[10] J.D. Denton and W.N. Dawes. Computational Fluid Dynamics for Turbomachinery Design. In Developments in Turbomachinery Design, John Denton editor, Professional Engineering Publishing, 1999.

[11] N. Gourdain, S. Burguburu, G.J. Michon, N. Ouayahya, F. Leboeuf and S. Plot. About the Numerical Simulation of Rotating Stall Mechanisms in Axial Compressors, ASME Paper GT2006-90223, 2006.

[12] C. Hirsch and R.P. Dring. Throughflow Models for Mass and Momentum-Averaged Variables. Transactions of the ASME Journal of Turbomachinery, 109, 362370, 1987.

[13] C. Hirsch. Numerical Computation of Internal and External Flows. Wiley, 1988.

[14] J.H. Horlock and J.D. Denton. A review of Some Early Design Practice Using Computational Fluid Dynamics and a Current Perspective. Journal of Turbomachinery, 127, 513, 2005.

[15] C.M. Rhie, A.J. Gleixner, C.J. Fischberg and R.M. Zacharias. Development and Application of a Multistage Navier-Stokes Solver - Part I:Multistage Modeling Using Bodyforces and Deterministic Stresses. ASME Paper 95GT-342, 1995.

[16] W.B. Roberts, G.K. Serovy and D.M. Sandercock. Modeling the 3-D Flow Effects on Deviation Angle for Axial Compressor Middle Stages. Journal of Engineering for Gas Turbines and Power, 108, 1986.

[17] J.F. Simon and O. Léonard. A Throughflow Analysis Tool Based on the Navier-Stokes Equations. Proceedings of the 6th European Turbomachinery Conference, Lille, 2005.

[18] J.F. Simon and O. Léonard. Modeling of 3-D Losses and Deviations in a Throughflow Analysis Tool. Journal of Thermal Science, Vol 16, No 3, pp 208-214, 2007.

[19] J.F. Simon. Contribution to Throughflow Modelling for Axial Flow Turbomachines. Ph.D. thesis, University of Liège, 2007.

[20] L.H. Smith, Jr. The Radial-Equilibrium Equation of Turbomachinery. Journal of Engineering for Power, January, 1966.

[21] A. Spurr. The Prediction of 3-D Transonnic Flow in Turbomachinery Using a Combined Throughflow and Bladeto-Blade Time Marching Method. Int. Journal of Heat and Fluid Flow, 2(4), 189199, 1980.

[22] A. Sturmayr. Evolution of a 3-D Structured Navier-Stokes Solver Towards Advanced Turbomachinery Applications. Ph.D. thesis, Vrije Universiteit Brussel, 2004.

[23] J.P. Thomas, J.F. Simon and O. Léonard. Investigating Circumferential Non Uniformities in Throughflow Calculations Using an Harmonic Reconstruction, ASME Paper GT2008-50328, 2008. 